\title{
ODS en el Derecho Financiero y Tributario en la Universidad del País Vasco-Euskal Herriko Unibertsitatea.
}

\author{
Irune Suberbiola Garbizu ${ }^{\mathrm{a}}$
}

aUniversidad del País Vasco-Euskal Herriko Unibertsitatea, irune.suberbiola@ehu.eus.

\begin{abstract}
In addition to instruments for capturing the resources needed to sustain public expenditures, taxes stand as tools of economic and social policy whose purpose is to meet the principles contained in our Constitution. The spirit of the social and democratic State of Law that advocates in its first article as superior values of the legal system freedom, justice, equality and political pluralism, and that is built in the Third Chapter of its Title I, coincides with the most part of the Sustainable Development Goals contained in the document "Transforming our world: the 2030 Agenda for Sustainable Development" adopted within the framework of the United Nations in 2015. This paper analyzes the role of the subject Financial and Tax Law in the incorporation of these SDGs, on the one hand, through the relevance of the role of taxes as tools of economic and social policy, emphasizing not only the relevance of more generalist taxes but also those of extra-fiscal taxes, and, on the other, empowering and mobilizing the students by providing them with an academic education to implement SDG solutions through the knowledge and skills acquired.
\end{abstract}

Keywords: ODS Objectives, Financial and Tax Law, extra-fiscal taxes, future training executors.

\footnotetext{
Resumen

Los tributos, además de instrumentos para la captación de los recursos necesarios para el sostenimiento de gastos públicos se erijen como herramientas de política económica y social cuya finalidad consiste en atender a realización de los principios contenidos en la Constitución. El espíritu del Estado social y democrático de Derecho que propugna en su artículo primero como valores superiores del ordenamiento jurídico la libertad, la justicia, la igualdad y el pluralismo político, y que se construye en el Capitulo Tercero de su Título I, coincide con el de la mayoría de los Objetivos de Desarrollo Sostenible contenidos en el documento "Transformando nuestro mundo: la Agenda 2030 para el Desarrollo Sostenible" adoptado en el marco de Naciones Unidas en 2015. Esta comunicación analiza el papel de la asignatura Derecho Financiero y Tributario en la incorporación de esos ODS, por un lado, a través de la puesta en relevancia del papel de los tributos como herramientas de política económica y social, subrayando no sólo la relevancia de los impuestos más
} 
generalistas sino también de los de corte extrafiscal, y, por otro, empoderando y movilizando al alumnado proporcionándole una formación académica para implementar soluciones ODS a través de los conocimientos y habilidades adquiridos.

Palabras clave: Objetivos ODS, Derecho Financiero y Tributario, tributos extrafiscales, formación futuros ejecutores.

\section{Introducción}

El Derecho Financiero y Tributario es la rama del Derecho Público que se ocupa del estudio de la actividad financiera de los entes públicos, la dirigida a la obtención, gestión y gasto de los recursos necesarios para atender la satisfacción de las necesidades colectivas. Usualmente la materia se divide en dos cursos; una parte general, donde se estudian los instituciones comunes a toda la actividad financiera pública, la caracterización de los principios constitucionales del ordenamiento financiero, y las reglas para el establecimiento y aplicación de las normas financieras; y una parte especial, donde se analizan los distintos tributos que componen nuestro sistema impositivo.

En la Universidad del País Vasco-Euskal Herriko Unibertsitatea, esa parte especial, la asignatura Derecho Financiero II, se imparte en el $4^{\circ}$ curso del Grado en Derecho y del Doble Grado en Derecho y ADE. La inciativa analizada en estas líneas se desarrolla, precisamente, con los grupos de euskara y castellano que cursan esta doble titulación; en total 60 alumnos/as con un alto nivel de autoexigencia, resultados académicos y participación en el aula y una prometedora proyección profesional en puestos de relevancia tanto en la empresa privada como en el ámbito público. Otra de las características que afecta a la docencia en estos grupos es que por una extraña configuración de su plan de estudios, el alumnado cursa la parte especial de la disciplina sin tener conocimientos previos de la parte general, lo cual obliga a la adaptar el temario y las actividades propuestas para que nuestros estudiantes puedan tener una visión global del panorama tributario patrio.

En este contexto, la inciativa que se presenta en esta comunicación imbrica los fines propios de la asignatura con los Objetivos de Desarrollo Sostenible contenidos en el documento "Transformando nuestro mundo: la Agenda 2030 para el Desarrollo Sostenible," adoptado en el marco de Naciones Unidas en septiembre de 2015 y que entró en vigor el 1 de enero de 2016. En concreto, las actividades realizadas presentan una innovación respecto a la práctica docente que precedía en la impartición de la asignatura en el sentido de que más allá de presentar el papel de los tributos como instrumentos recaudatorios subraya su papel como herramientas de política económica y social que 
pueden coadyuvar en las aspiraciones ODS, que para el año 2030 hayamos situado al mundo y a sus sociedades en un camino hacia un futuro mejor.

En efecto, los tributos, además de instrumentos para la captación de los recursos necesarios para el sostenimiento de gastos públicos se erijen como herramientas de política económica y social con la finalidad de atender a realización de los principios contenidos en la Constitución, una Constitución cuyo artículo primero impulsa el espíritu del Estado social y democrático de Derecho propugnando como valores superiores del ordenamiento jurídico la libertad, la justicia, la igualdad y el pluralismo político. Pues bien, estos valores se construyen mediante los principios instituidos en el Capítulo Segundo (derecho a la igualdad, vida, libertad y seguridad, honor...) y principalmente Tercero de su Título I, protección social, económica y jurídica de la familia; progreso social y económico; una distribución de la renta regional y personal más equitativa; protección de la salud, cultura medio ambiente adecuados; participación libre y eficaz de la juventud en el desarrollo político, social, económico y cultura; suficiencia económica de la tercera edad,... Como podemos observar estos principios coinciden con muchos (si no la mayoría) de los 17 Objetivos de Desarrollo Sostenible contenidos en la Agenda $2030^{1}$, fin del hambre, seguridad alimentaria, igualdad, educación, crecimiento económico sostenido,reducción de desigualdades, protección del medioambiente..., lo cual permite incorporarlos en la docencia de la asignatura para poner en relavancia el papel que pueda jugar el Derecho Financiero y Tributario, y más concretamente los tributos, en la consecución de los mismos $^{2}$.

Con esta finalidad, se plantean una serie de iniciativas para que el alumnado adquiera consciencia de la relevancia de los tributos no sólo desde una perspectiva recaudatoria, sino, también, desde su papel como herramientas que impulsan el cambio social. Por cuestiones de tiempo y espacio, haremos referencia únicamente a una de las acciones realizadas a lo largo del desarrollo de la asignatura, en el entendimiento de que dichas acciones se encuadran en un plano cronológico y temático que abarca más actividades en relación con los objetivos perseguidos. Pasemos, pues, a analizar dichos objetivos, el desarrollo de la innovación propuesta, los resultados obtenidos, $\mathrm{y}$, finalmente las conclusiones colegidas de la experiencia de los dos últimos cursos en los que se ha comenzado desarrollar la incorporación de los ODS en el ámbito de la enseñanza del Derecho Financiero en el Doble Grado Derecho-ADE de la Universidad del País VascoEuskal Herriko Unibertsitatea.

\footnotetext{
${ }^{1}$ NACIONES UNIDAS, Sustinable Development Goals, https://sustainabledevelopment.un.org/?menu=1300, [Consulta: 7 de marzo de 2019] ; REDS, Cómo Empezar con los ODS en las Universidades, http://reds-sdsn.es/wp-content/uploads/2017/02/GuiaODS-Universidades-1800301-WEB.pdf. [Consulta: 3 de febrero de 2019].

${ }^{2}$ Ver al respecto, entre otros GARCÍA CALVENTE, Y.; RUIZ GARIJO, M. "La necesidad de un Derecho Financiero Social como respuesta a la ciris financiera" en Adame Martínez, F. Ramos Prieto, J.; Lasarte Álvarez, J. Estudios sobre el sistema tributario actual y la situación financiera del sector público: Homenaje al Profesor Dr. D. Javier Lasarte Álvarez, Madrid: INstituto de Estudios Fiscales.
} 


\section{Objetivos}

Dentro de los propósitos perseguidos, el punto de partida en la incorporación de los ODS en el marco de la asignatura Derecho Financiero II es, en primer lugar, el propio conocimiento y comprensión del marco de los ODS, de su propósito y de sus aplicaciones, y el de las implicaciones del papel que los tributos pueden desarrollar en la ejecución de los mismos.

Existen también otros objetivos que afloran de forma tangencial, pero no menos importante, y que se adhieren a las competencias perseguidas por la asignatura, como son que los/las estudiantes adquieran las destrezas y habilidades transversales necesarias para su implementación, esto es, que desarrollen un pensamiento crítico y sistémico en la resolución integral de problemas relacionados con los ODS, y que adquieran competencias normativas, estratégicas y de colaboración, al tiempo que desarrollan su creatividad, habilidades de aprendizaje, competencias de asociación, responsabilidad social y medioambiental. En este sentido, estos objetivos secundarios se entrelazan con los recursos y herramientas específicos para la consecución de los objetivos de aprendizaje propios de la disciplina y, en su caso, las pruebas de conocimiento o las tareas y actividades encomendadas para su plasmación.

Un tercer objetivo, éste sí subsidiario o no al menos tan importante o prioritario como los precedentes, es el de sembrar la semilla que impregne el espíritu ODS en futuros ejecutores y responsables de la Agenda 2030. El perfil de los estudiantes del Doble Grado en DerechoADE, su potencial desarrollo profesional, permite que mediante estas iniciativas se origine el desarrollo de liderazgos que orienten a los ODS la ulterior actividad empresarial o institucional de nuestros/as alumnos/as.

\section{Desarrollo de la innovación}

Como comentábamos en la introducción a esta comunicación, la innovación descrita en la misma forma parte de un conjunto de actividades que tienen por objeto la lectura del Derecho Financiero y Tributario desde el prisma de los ODS. Las actividades se han desarrollado en los grupos del Doble Grado ADE-Derecho y como grupo de control se ha utilizado el grupo de euskara del curso 2018-2019 del Grado en Derecho, donde el hecho de que se comparta la asignatura entre varios profesores ha imposibilitado llevar a cabo la iniciativa. Así si bien la innovación se ha desplegado a lo largo de los cursos 2017-2018 y 2018-2019, los resultados reflejados en la misma son los recogidos en el punto 4 teniendo en cuenta las opiniones esgrimidas por el alumnado de la Facultad de Ciencias Empresariales (donde se cursa el Doble grado) y el que conforma el grupo en euskara de la Facultad de Derecho, Sección Vizcaya en este último curso.

Razones de tiempo y espacio nos obligan a limitarnos a la descripción de una única actividad de las propuestas a los/las estudiantes. Sin embargo, y para enmarcar el desarrollo que el tratamiento de los ODS tiene en la asignatura, pasamos brevemente a retratar las actividades realizadas en en el desarrollo de su programa indicando, en su caso, los objetivos/implicaciones ODS que se derivan de cada una de ellas en el la Tabla 1. 
Tabla 1. Unidades didácticas, actividades propuestas y objetivos/implicación ODS

\begin{tabular}{|c|c|c|}
\hline $\begin{array}{c}\text { UNIDAD } \\
\text { DIDÁCTICA/MÓDULO }\end{array}$ & ACTIVIDAD PROPUESTA & OBJETIVO/IMPLICACIÓN ODS \\
\hline $\begin{array}{l}\text { Aspectos básicos del Derecho } \\
\text { Financiero y Tributario }\end{array}$ & $\begin{array}{l}\text { Mapa conceptual de los entes con } \\
\text { poder tributario }\end{array}$ & $\begin{array}{l}\text { Identificación de los actores } \\
\text { institucionales con competencias en la } \\
\text { materia }\end{array}$ \\
\hline El tributo & $\begin{array}{l}\text { Análisis de los tributos desde la } \\
\text { perspectiva de los principios de } \\
\text { justicia tributaria }\end{array}$ & $\begin{array}{l}\text { Lectura crítica de un tributo desde la } \\
\text { perspectiva ODS. }\end{array}$ \\
\hline La relación jurídico tributaria & $\begin{array}{l}\text { Creación de un tributo con } \\
\text { finalidad extrafiscal }\end{array}$ & Cualquiera de los 17 objetivos ODS. \\
\hline $\begin{array}{l}\text { Procedimientos de aplicación } \\
\text { de los tributos }\end{array}$ & Resolución de un caso práctico & \\
\hline $\begin{array}{l}\text { Ilícitos tributarios } \\
\text { procedimiento sancionador }\end{array}$ & Análisis de una noticia & $\begin{array}{l}\text { Análisis de una noticia desde la } \\
\text { perspectiva de la responsabilidad social } \\
\text { corporativa en materia tributaria }\end{array}$ \\
\hline $\begin{array}{l}\text { Recursos y reclamaciones } \\
\text { contra la Hacienda }\end{array}$ & $\begin{array}{l}\text { Linea del tiempo con las distintas } \\
\text { posibilidades/alternativas que } \\
\text { confluyen en los procedimientos de } \\
\text { aplicación de los tributos, el } \\
\text { procedimiento sancionador y los } \\
\text { procedimientos de revisión. }\end{array}$ & \\
\hline $\begin{array}{l}\text { Fiscalidad Directa IRPF y } \\
\text { Patrimonio }\end{array}$ & $\begin{array}{l}\text { Análisis crítico del impuesto, } \\
\text { especial referencia a los beneficios } \\
\text { fiscales. }\end{array}$ & $\begin{array}{l}\text { Análisis de las exenciones, } \\
\text { bonificaciones, reducciones y } \\
\text { deducciones del impuesto a la luz de } \\
\text { los } 17 \text { objetivos ODS. Presentación de } \\
\text { alternativas. }\end{array}$ \\
\hline Fiscalidad Directa IS & $\begin{array}{l}\text { Análisis critico del impuesto, } \\
\text { especial referencia a los beneficios } \\
\text { fiscales. }\end{array}$ & $\begin{array}{l}\text { Análisis de las exenciones, } \\
\text { bonificaciones, reducciones y } \\
\text { deducciones del impuesto a la luz de } \\
\text { los } 17 \text { objetivos ODS. Presentación de } \\
\text { alternativas. }\end{array}$ \\
\hline Fiscalidad Directa ISD & $\begin{array}{l}\text { Análisis crítico del impuesto, } \\
\text { especial referencia a los beneficios } \\
\text { fiscales. }\end{array}$ & $\begin{array}{l}\text { Análisis de las exenciones, } \\
\text { bonificaciones, reducciones y } \\
\text { deducciones del impuesto a la luz de } \\
\text { los } 17 \text { objetivos ODS. Presentación de } \\
\text { alternativas. }\end{array}$ \\
\hline Fiscalidad Indirecta IVA & $\begin{array}{l}\text { Análisis crítico del impuesto, } \\
\text { especial referencia a los beneficios } \\
\text { fiscales. }\end{array}$ & $\begin{array}{l}\text { Análisis de las exenciones, } \\
\text { bonificaciones, reducciones y } \\
\text { deducciones del impuesto a la luz de } \\
\text { los } 17 \text { objetivos ODS. Presentación de } \\
\text { alternativas. }\end{array}$ \\
\hline Fiscalidad Indirecta ITPAJD & $\begin{array}{l}\text { Análisis crítico del impuesto, } \\
\text { especial referencia a los beneficios } \\
\text { fiscales. }\end{array}$ & $\begin{array}{l}\text { Análisis de las exenciones, } \\
\text { bonificaciones, reducciones y } \\
\text { deducciones del impuesto a la luz de } \\
\text { los } 17 \text { objetivos ODS. Presentación de } \\
\text { alternativas. }\end{array}$ \\
\hline Impuestos especiales & $\begin{array}{l}\text { Análisis crítico del impuesto, } \\
\text { especial referencia a los beneficios } \\
\text { fiscales. }\end{array}$ & $\begin{array}{l}\text { Análisis de las exenciones, } \\
\text { bonificaciones, reducciones y } \\
\text { deducciones del impuesto a la luz de } \\
\text { los } 17 \text { objetivos ODS. Presentación de } \\
\text { alternativas. }\end{array}$ \\
\hline
\end{tabular}

Fuente: Elaboración propia

Dentro de las distintas unidades didácticas recogidas en la tabla precedente, nos referiremos ahora a la actividad contenida en la unidad titulada "la relación jurídico tributaria", donde partiendo del esquema de liquidación ideal de todo tributo, los alumnos deben crear un tributo extrafiscal (tasa, contribución especial o impuesto) que tenga como finalidad fomentar la consecunción de uno de los 17 objetivos ODS. En las dos últimas ediciones de la iniciativa, los estudiantes se han decantado por la generación de impuestos con un claro sesgo medioambiental, acordando, en primer lugar (2017-2018), la creación de tributos que penalicen la generación de residuos sólidos y, en segunda instancia (2018-2019) la de aquellos que propicien la no generación de gases contaminantes.

Para el desarrollo de la actividad la clase se divide por parte del profesorado en distintos grupos lo suficientemente heterogéneos atendiendo a las particularidades personales que cada alumno/a ha reflejado en un Test de Hermann previamente realizado. Con ello se pretende que los alumnos se acostumbren a colaborar con compañeros que no tienen por qué compartir sus modos y usos de trabajo. 
Una vez presentados los 17 objetivos ODS de la Agenda 2030 y las características y retos de cada uno de ellos, el aula en su conjunto, a través de los representantes de los distintos grupos, decide el objetivo a perseguir por los tributos que van a ser diseñados singularmente pero de forma conjunta, como decíamos en las ediciones anteriores la no generación de residuos sólidos o gases contaminantes.

Tras la definición del objetivo general, cada grupo debe diseñar la estrategia fiscal que materialice dicho objetivo en la configuración de un tributo que recoja la estructura general de toda tasa, impuesto o contribución especial, atendiendo a los elementos que componen la relación jurídico-tributaria, los principios tributarios de justicia material así como los aspectos más esenciales de la forma tributaria elegida. Para ello al grupo se le exige que redacte el articulado del tributo diseñado y que represente dicho articulado en un póster que contenga los elementos esenciales del tributo en el esquema de liquidación resultante de dicho articulado.

El tiempo estimado para la realización de la tarea encomendada se extiende a las cuatro horas de las cuales la primera, a modo de preparación se desarrolla en la propia aula de suerte que el alumnado pueda 1) elegir el ODS global, 2) consensuar la rúbrica por la que la actividad será evaluada, 3) esbozar la configuración de la tasa, impuesto o contribución especial a delinear, y 4) analizar si de la misma se infieren los objetivos de aprendizaje específicos exigidos en la tarea así como el cumplimiento del ODS seleccionado. Las siguientes dos horas necesarias para la consumación de la tarea se desarrollan en grupo, pero fuera del aula, de suerte que se pueda proceder en un entorno más propicio, a la redacción del articulado y a la confección del póster requerido. Finalmente, la cuarta hora de la tarea se desenvuelve de nuevo en el aula y es utilizada para la pertinente presentación del artículado y póster por los miembros del grupo así como para su evaluación por parte del resto de compañeros atendiendo a la rúbrica común previamente acordada.

Respecto a los materiales y herramientas utilizados para la actividad además de los propios del módulo o unidad didáctica (aquellos facilitados en el aula virtual para una mejor comprensión de los conceptos, teorías...), para la redacción del articulado se requiere un procesador de textos (a elección del alumnado), siendo indiferente la herramienta utilizada para la confección del póster/infogracía que ejemplifique el esquema de liquidación del tributo diseñado (ease.ly, genial.ly, piktochart o incluso pinturas, papel y tijeras). 


\begin{tabular}{|c|c|}
\hline Tipo de actividad & Actividad de Aprendizaje 2, diseño de un tributos con ODS \\
\hline Deseripción & $\begin{array}{l}\text { Redacción del articulado de un tributo que recoja la estructura de liquidación general de un } \\
\text { impuesto atendiendo a los elementos que componen la relación juridico tributaria, los } \\
\text { principios tributarios de justicia material así como los aspectos más esenciales del } \\
\text { concepto. Los alumnos también deben presentar un póster/infografia con la estructura del } \\
\text { tributo. }\end{array}$ \\
\hline \multirow{4}{*}{$\begin{array}{ll}\text { Objetivo de } \\
\text { aprendizaje de la } \\
\text { asignatura }\end{array}$} & $\begin{array}{l}\text { OA 2: Identificar los principios fínancieros y tributarios contenidos en nuestro } \\
\text { ordenamiento. }\end{array}$ \\
\hline & OA 3: Identificar el concepto, clase y estructura de los tributos. \\
\hline & $\begin{array}{l}\text { OA 4: Identificar los elementos objetivos, subjetivos, espaciales, temporales y } \\
\text { cuantitativos derivados de la relación jurídico-tributaria. }\end{array}$ \\
\hline & $\begin{array}{l}\text { OA 10: Adquisición de habilidades (orales y escritas) para la exposición de trabajos, con } \\
\text { profesionalidad y con la oportuna sensibilidad que requiere la materia. }\end{array}$ \\
\hline \multirow[t]{8}{*}{ Objetivo ODS } & $\begin{array}{l}\text { El conocimiento y comprensión del marco de los ODS, de su propósito y de sus } \\
\text { aplicaciones. }\end{array}$ \\
\hline & $\begin{array}{l}\text { Reflexionar sobre las implicaciones del papel que los tributos pueden desarrollar en la } \\
\text { ejecución de los mismos. }\end{array}$ \\
\hline & Profundizar en el \\
\hline & $\begin{array}{l}\text { conocimiento de uno cualquiera de ellos, elegido entre los } 17 \text { ODS contemplados en la } \\
\text { Agenda } 2030 \text {. }\end{array}$ \\
\hline & $\begin{array}{l}\text { Desarrollar un pensamiento crítico y sistémico en la resolución integral de problemas } \\
\text { relacionados con los ODS }\end{array}$ \\
\hline & Adquirir de competencias normativas, estratégicas y de colaboración. \\
\hline & $\begin{array}{l}\text { Desarrollar la creatividad, habilidades de aprendizaje, competencias de asociación, } \\
\text { responsabilidad social y medioambiental del alumnado. }\end{array}$ \\
\hline & $\begin{array}{l}\text { Construcción del espíitu el espíritu ODS en el alumnado como futuros ejecutores y } \\
\text { responsables de la Agenda } 2030 .\end{array}$ \\
\hline $\begin{array}{l}\text { Materiales } \\
\text { necesarios }\end{array}$ & $\begin{array}{l}\text { Además de los propios del módulo como herramienta un procesador de textos. Es } \\
\text { indiferente el la herramienta utilizada para la confección del pósterinfografia (Easel.ly, } \\
\text { Genial.ly; Piktochart... o cualquier otra). }\end{array}$ \\
\hline \multirow[t]{3}{*}{ Cronograma } & $\begin{array}{l}\text { 1.h. Preparación en el aula: } \\
\text { - Elección del ODS global. } \\
\text { - Consensuar la rúbrica por la que la actividad será evaluada. } \\
\text { - Esbozar la configuración de la tasa, impuesto o contribución especial a delinear. } \\
\text { - Analizar si de la misma se infieren los objetivos de aprendizaje específicos } \\
\text { exigidos en la tarea así como el cumplimiento del ODS seleccionado. }\end{array}$ \\
\hline & 2-3 h. Trabajo finera del aula, redacción del articulado y confección del poster. \\
\hline & $\begin{array}{l}4 \text { h. Presentación y evaluación en el aula de la presentación a través de la rúbrica } \\
\text { consensuada en la primera hora. }\end{array}$ \\
\hline Evaluación & Aplicación de la rúbrica consensuada en clase. \\
\hline
\end{tabular}

Fuente: elaboración propia

\section{Resultados}

Los resultados obtenidos con la innovación propuesta no pueden ser más enriquecedores. Los/as alumnos/as han referido que esta actividad no sólo les ha permitido afianzar los resultados de aprendizaje propios de la asignatura, sino que la introducción de la perspectiva extrafiscal de los tributos, en concreto la aportada por la Agenda 2030, les ha permitido desarrollar un espíritu crítico y constructivo respecto al papel que los mismos puedan jugar en la consecución de los ODS.

Los ejemplos recogidos en las siguientes fotografías da muestra del elevado grado de implicación con el que los alumnos se han consagrado a la tarea, tanto desde un punto de vista formal, como desde un punto de vista material. 


\section{Ley sobre el Impuesto de Calderas de Carbón Domésticas}

Introducción

En la presente práctica vamos a desarrollar un tributo que estará enfocado además de a fines recaudatorios a conseguir minimizar la emisión de gases contaminantes, en este caso concreto el dióxido de azufre. La forma de llevar esto a cabo será mediante la creación de una Ley que diseñará un impuesto y una contribución especial que incidirán en el uso de este contaminante en calderas de carbón domésticas.

Las principales fuentes de emisión de dióxido de azufre a la atmósfera son la combustión de productos petrolíferos y la quema de carbón en centrales eléctricas y calefacciones centrales junto a otras muy diversas. Debido a esto hay que remarcar el efecto contaminante que produce el continuo uso de calderas de carbón domésticas y la necesidad de fomentar el cambio de éstas por medios más sostenibles con el medioambiente.

\section{Preámbulo}

I

La presente Ley tiene como objetivo armonizar nuestro sistema fiscal con un uso más eficiente y respetuoso con el medioambiente y la sostenibilidad, valores que inspiran esta reforma de la fiscalidad, y como tal en línea con los principios básicos que rigen la política fiscal y ambiental de la Unión Europea.

En la sociedad actual, la incidencia, cada vez mayor de los diversos contaminantes perjudiciales para el medio ambiente requiere de un marco normativo y regulatorio que garantice un uso racional y controlado de los mismos con el claro objetivo de compaginar la vida social con la sostenibilidad ambiental.

El fundamento básico de esta Ley reside en el artículo 45 de la Constitución, precepto en el que la protección de nuestro medio ambiente se configura como uno de los principios rectores de las políticas sociales y económicas. Por ello, uno de los ejes de esta reforma tributaria será la internalización de los costes medioambientales derivados de la combustión de gases de las calderas de carbón. De esta forma, la ley ha de servir de estímulo para mejorar nuestros niveles de eficiencia medioambiental a la vez que permiten asegurar una mejor gestión de los recursos naturales y seguir avanzando en el nuevo modelo de desarrollo sostenible, tanto desde el punto de vista económico y social, como medioambiental.

La presente reforma contribuye además a la integración de las políticas medioambientales en nuestro sistema tributario, en el cual tienen cabida tanto tributos específicamente ambientales, como la posibilidad de incorporar el elemento ambiental en otros tributos ya existentes.

A tal fin, mediante esta Ley se regula un nuevo impuesto y a su vez una contribución especial en el futuro: el impuesto sobre calderas de carbón domésticas y la contribución especial relativa al cambio de calderas de carbón domésticas; se pretende modificar los tipos impositivos establecidos para el carbón, creándose además exenciones y

Fig. 1. Ejemplo de redacción de artículado de tributo con ODS realizado por alumnos/as 


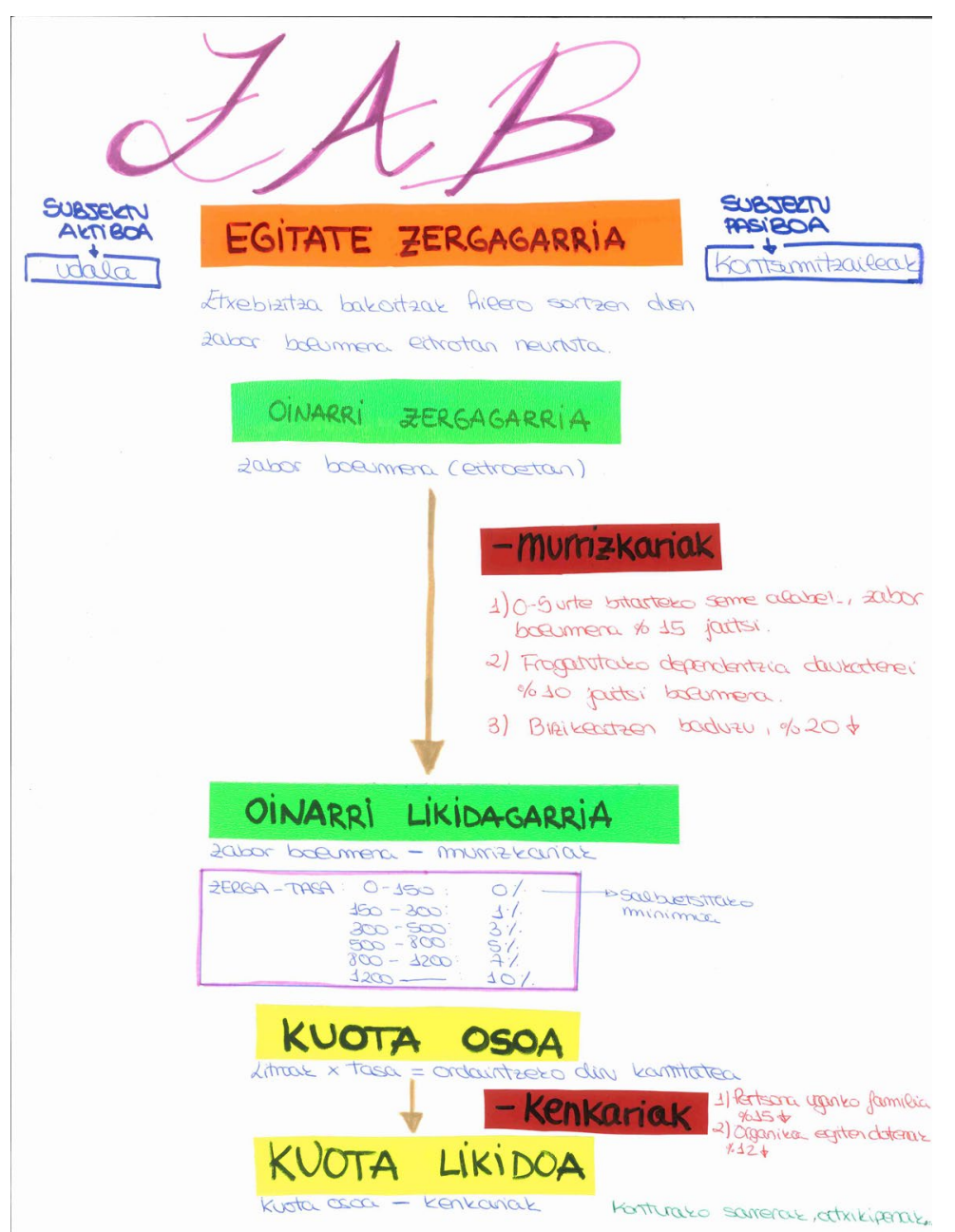

\section{KUOTA DIFERENTZIALA}

Fig. 2. Ejemplo de esquema de liquidación de tributo con ODS realizado por alumnos/as

Al conjugar las sensaciones manifestadas por los alumnos con los resultados plasmados en las evaluaciones realizadas sobre los mismos, se aprecia que esta iniciativa no sólo abunda en el conocimiento de los ODS recalcando el papel de los tributos como instrumentos de política económica y social, sino, también, en el espíritu crítico con el que el alumnado se enfrenta a las actividades que, según la Tabla 1, realizará con posterioridad. En este sentido la experiencia es importante puesto que el análisis comparativo de la docencia ofrecida en el Grado en Derecho y el Doble Grado en Derecho y ADE de la Universidad del País Vasco ha demostrado que los alumnos del primero no sólo tienen limitaciones a la hora de reconocer las finalidades extrafiscales de los tributos, aquellas que no son meramente recaudatorias, sino que poseen un total desconocimiento de los objetivos marcados en la "Agenda 2030 sobre el Desarrollo Sostenible de Naciones Unidas". 
Los resultados de la encuestas realizadas al respecto en los grupos de Doble Grado ADEDerecho y el grupo de euskara del Grado en Derecho en diciembre de 2018 y recogidos en la Tabla 3, a continuación, son meridianos en cuanto a la necesidad de introducir la perspectiva de los ODS en la enseñanza del Derecho Financiero y Tributario en la Universidad.

Tabla 3. Encuesta sobre ODS - Derecho Financiero y Tributario diciembre 2018

\begin{tabular}{|c|c|c|}
\hline & $\begin{array}{l}\text { ALUMNADO DOBLE } \\
\text { GRADO }\end{array}$ & $\begin{array}{l}\text { ALUMNADO GRADO EN } \\
\text { DERECHO }\end{array}$ \\
\hline $\begin{array}{c}\text { Número de alumn@s } \\
\text { encuestad@s/matriculad } @ \text { s }\end{array}$ & $47 / 50$ & $22 / 35$ \\
\hline ¿Sabes lo que son los ODS? & $\begin{array}{l}\text { Si: } 43 \\
\text { No: } 2 \\
\text { Ns/nc: } 2\end{array}$ & $\begin{array}{c}\text { Si: } 2 \\
\text { No: } 18 \\
\text { Ns/nc: } 2\end{array}$ \\
\hline $\begin{array}{c}\text { ¿Conoces el papel que tiene el } \\
\text { Derecho Financiero y Tributario } \\
\text { en su desarrollo? }\end{array}$ & $\begin{array}{l}\text { Si: } 43 \\
\text { No: } 2 \\
\text { Ns/nc: } 2\end{array}$ & $\begin{array}{c}\text { Si: } 2 \\
\text { No: } 18 \\
\text { Nsinc: } 2\end{array}$ \\
\hline $\begin{array}{l}\text { ¿Las actividades realizadas en la } \\
\text { asignatura te han servido para } \\
\text { afianzar tus conocimientos sobre } \\
\text { la materia? }\end{array}$ & $\begin{array}{c}\text { Si: } 43 \\
\text { No: } 1 \\
\text { Ns/nc: } 3\end{array}$ & $\begin{array}{c}\text { Si: } 0 \\
\text { No: } 18 \\
\text { Ns/nc: } 4\end{array}$ \\
\hline $\begin{array}{l}\text { ¿Ha cambiado tu perspectiva y } \\
\text { actitud sobre la visión de los } \\
\text { ODS? }\end{array}$ & $\begin{array}{l}\text { Si: } 43 \\
\text { No: } 2 \\
\text { Ns/nc: } 2\end{array}$ & $\begin{array}{c}\text { Si: } 0 \\
\text { No: } 18 \\
\text { Nsinc: } 4\end{array}$ \\
\hline $\begin{array}{l}\text { ¿Y respecto al Derecho } \\
\text { Financiero y Tributario? }\end{array}$ & $\begin{array}{c}\text { Si: } 43 \\
\text { No: } 2 \\
\text { Ns/nc: } 2\end{array}$ & $\begin{array}{c}\text { Si: } 0 \\
\text { No: } 18 \\
\text { Ns/ne: } 4\end{array}$ \\
\hline $\begin{array}{l}\text { En el futuro, ¿te planteas una } \\
\text { actitud más crítica/constructiva } \\
\text { sobre los ODS? }\end{array}$ & $\begin{array}{c}\text { Si: } 43 \\
\text { No: } 2 \\
\text { Ns/nc: } 2\end{array}$ & $\begin{array}{c}\text { Si: } 0 \\
\text { No: } 18 \\
\text { Nsinc: } 4\end{array}$ \\
\hline
\end{tabular}

\section{Conclusiones}

Las conclusiones que podemos inferir de la experiencia desarrollada a lo largo de estos dos últimos cursos, y presentada como innovación docente en esta comunicación, son acordes a los resultados referidos en el epígrafe precedente.

La enseñanza del Derecho Financiero y Tributario, por el papel preeminente que juegan los tributos como instrumentos de política económica y social, resulta un elemento catalizador para la introducción de los 17 ODS contenidos en la Agenda 2030. Más aún, las iniciativas planteadas abundan en la adquisición de competencias transversales íntimamente ligadas a las propias de la asignatura y a las consustanciales en los objetivos del Desarrollo Sostenible planteados por Naciones Unidas.

En este sentido, a futuro, desde el área de Derecho Financiero y Tributario del Departamento de Derecho Público de la Universidad del País Vasco, y en línea con lo propuesto por la $\mathrm{CRUE}^{3}$, se plantea como necesaria la extensión de esta iniciativa más allá del Doble Grado ADE-Derecho a los alumnos de ciencias jurídicas no sólo en lo que atañe a nuestra disciplina sino también a asignaturas afines que pueda tener alguna implicación en

3 CRUE, Las Universidade acuerdan su contribución al plan de acción de la Agenda 2030, http://www.crue.org/Comunicacion/Noticias/Las $\% 20$ universidades $\% 20$ acuerdan $\% 20$ su $\% 20$ contribución $\% 20$ al $\% 20 \mathrm{Plan} \% 20 \mathrm{de} \% 2$ 0Acción\%20de\%20la\%20Agenda\%202030.aspx. [Consulta: 10 de marzo de 2019] 
la formación de las personas que, en un futuro no muy lejano se hagan cargo profesional o personalmente del desarrollo de este tipo de objetivos ODS en sus distintos proyectos.

\section{Referencias}

CRUE, Las Universidade acuerdan su contribución al plan de acción de la Agenda 2030, http://www.crue.org/Comunicacion/Noticias/Las\%20universidades $\% 20$ acuerdan $\% 20$ su $\% 2$ 0contribución\%20al\%20Plan\%20de\%20Acción\%20de\%20la\%20Agenda\%202030.aspx. [Consulta: 10 de marzo de 2019]

GARCÍA CALVENTE, Y.; RUIZ GARIJO, M. "La necesidad de un Derecho Financiero Social como respuesta a la ciris financiera" en Adame Martínez, F. Ramos Prieto, J.; Lasarte Álvarez, J. Estudios sobre el sistema tributario actual y la situación financiera del sector público: Homenaje al Profesor Dr. D. Javier Lasarte Álvarez, Madrid: INstituto de Estudios Fiscales.

NACIONES UNIDAS, Sustinable Development Goals, https://sustainabledevelopment.un.org/?menu=1300 [Consulta: 7 de marzo de 2019]

REDS, Cómo Empezar con los ODS en las Universidades, http://reds-sdsn.es/wpcontent/uploads/2017/02/Guia-ODS-Universidades-1800301-WEB.pdf. [Consulta: 3 de febrero de 2019] 